\title{
Can LV Dyssynchrony as Assessed with Phase Analysis on Gated Myocardial Perfusion SPECT Predict Response to CRT?
}

\author{
Maureen M. Henneman ${ }^{1}$, Ji Chen ${ }^{2}$, Petra Dibbets-Schneider ${ }^{3}$, Marcel P. Stokkel ${ }^{3}$, Gabe B. Bleeker ${ }^{1,4}$, Claudia Ypenburg ${ }^{1}$, \\ Ernst E. van der Wall ${ }^{1,4}$, Martin J. Schalij ${ }^{1}$, Ernest V. Garcia ${ }^{2}$, and Jeroen J. Bax ${ }^{1}$ \\ ${ }^{I}$ Department of Cardiology, Leiden University Medical Center, Leiden, The Netherlands; ${ }^{2}$ Department of Radiology, Emory University \\ School of Medicine, Atlanta, Georgia; ${ }^{3}$ Department of Nuclear Medicine, Leiden University Medical Center, Leiden, The Netherlands; \\ and ${ }^{4}$ The Interuniversity Cardiology Institute of The Netherlands, Utrecht, The Netherlands
}

\begin{abstract}
Cardiac resynchronization therapy (CRT) is now a well-recognized therapeutic option for patients with end-stage heart failure. However, not all patients respond to CRT, and, therefore, preimplantation identification of responders is desirable. The aim of the present study was to investigate whether the degree of left ventricular (LV) dyssynchrony, as assessed with phase analysis from gated myocardial perfusion SPECT (GMPS), can predict which patients will respond to CRT. Methods: Forty-two patients with severe heart failure, depressed LV ejection fraction, and wide QRS complex were prospectively included for implantation of a CRT device and underwent GMPS and 2-dimensional echocardiography as part of the clinical protocol. Clinical status was evaluated using the New York Heart Association (NYHA) classification, 6-min walk test, and quality-of-life score. The histogram bandwidth and phase SD (parameters indicating LV dyssynchrony) were assessed from GMPS, and the clinical status and echocardiographic variables were reassessed at 6-mo followup. Results: Responders $(71 \%)$ and nonresponders $(29 \%)$ had comparable baseline characteristics, except for histogram bandwidth $\left(175^{\circ} \pm 63^{\circ}\right.$ vs. $\left.117^{\circ} \pm 51^{\circ}[P<0.01]\right)$ and phase $\mathrm{SD}\left(56.3^{\circ} \pm 19.9^{\circ}\right.$ vs. $\left.37^{\circ} .1 \pm 14.4^{\circ}[P<0.01]\right)$, which were significantly larger in responders compared with nonresponders. Moreover, receiver-operating-characteristic curve analysis demonstrated an optimal cutoff value of $135^{\circ}$ for histogram bandwidth (sensitivity and specificity of $70 \%$ ) and of $43^{\circ}$ for phase SD (sensitivity and specificity of $74 \%$ ) for the prediction of response to CRT. Conclusion: Response to CRT is related to the presence of $L V$ dyssynchrony assessed by phase analysis with GMPS. A cutoff value of $135^{\circ}$ for histogram bandwidth and of $43^{\circ}$ for phase SD could be used to predict response to CRT. Larger prospective studies are warranted to confirm the present findings.
\end{abstract}

Key Words: left ventricular dyssynchrony; cardiac resynchronization therapy; SPECT; heart failure

J Nucl Med 2007; 48:1104-1111

DOI: 10.2967/jnumed.107.039925

Received Jan. 17, 2007; revision accepted Mar. 23, 2007.

For correspondence or reprints contact: Jeroen J. Bax, MD, PhD, Department of Cardiology, Leiden University Medical Center, Albinusdreef 2, 2333 ZA Leiden, The Netherlands.

E-mail: jbax@knoware.nl

COPYRIGHT $\odot 2007$ by the Society of Nuclear Medicine, Inc.
I n the recent years, cardiac resynchronization therapy (CRT) has emerged as a new treatment strategy for a subgroup of patients with end-stage heart failure, a depressed left ventricular ejection fraction (LVEF), and wide QRS complex on the surface electrocardiogram $(>120 \mathrm{~ms})(1)$. Although initial studies reported promising results, approximately $20 \%-30 \%$ of the patients do not respond to CRT (1-4). Recent data have indicated that LV dyssynchrony may be mandatory for response to CRT $(5,6)$. A wide QRS complex may not adequately indicate LV dyssynchrony, as $30 \%$ of the patients with a wide QRS complex (>120 ms) do not have substantial LV dyssynchrony on echocardiography whereas, on the other hand, approximately one third of the patients with a narrow QRS appear to have substantial LV dyssynchrony on echocardiography $(7,8)$.

At present, various imaging techniques are available for the assessment of LV dyssynchrony, including echocardiography with tissue Doppler imaging (TDI), or strain imaging, and MRI $(9,10)$. Since the early 1980 s, the possibility of assessing dyssynchronous cardiac contraction with nuclear imaging has been explored. Most studies were performed with gated blood-pool ventriculography for evaluation of cardiac dyssynchrony. Although this technique allows assessment of LVEF in a very reliable manner $(11,12)$, it does not provide information on the presence of ischemia or myocardial infarction. Recently, a new technique was developed to allow phase analysis from gated myocardial perfusion SPECT (GMPS). GMPS is used for assessment of myocardial perfusion but also provides information on regional wall thickening $(13,14)$. A count-based method has been developed to extract amplitude (systolic wall thickening) and phase from the regional LV count changes throughout the cardiac cycle (15). The phase information is related to the time interval when a region in the 3-dimensional (3D) LV myocardial wall starts to contract. It provides information as to how uniform or inhomogeneous is the distribution of these time intervals for the entire LV - that is, a measure of LV (dys)synchrony (15). 
The purpose of this study was to investigate whether the degree of LV dyssynchrony, as assessed with phase analysis from GMPS, can predict which patients will respond to CRT.

\section{MATERIALS AND METHODS}

The study population consisted of 42 patients with heart failure who were prospectively included for implantation of a CRT device. Patients with previously implanted pacemakers were included. Selection criteria for CRT were severe heart failure (New York Heart Association [NYHA] class 3 or 4), depressed LVEF $(<35 \%)$, and prolonged QRS duration $(>120 \mathrm{~ms})$.

The study protocol was as follows: Before implantation of the CRT device, resting GMPS with ${ }^{99 \mathrm{~m}} \mathrm{Tc}$ tetrofosmin was performed to assess LV dyssynchrony using phase analysis (15).

Next, clinical status was evaluated, which included the assessment of NYHA functional class, 6-min walk test, and quality-oflife score (using the Minnesota Quality of Life Questionnaire) (16). In addition, 2-dimensional (2D) echocardiography was performed for the assessment of LV volumes and LVEF. Clinical status and echocardiographic variables were reassessed at 6-mo follow-up.

\section{Gated SPECT}

Assessment of LV dyssynchrony from GMPS using phase analysis was recently reported (15). GMPS imaging with ${ }^{99 \mathrm{~m}} \mathrm{Tc}$ tetrofosmin (500 MBq, injected at rest) was performed using a triple-head SPECT camera system (GCA 9300/HG; Toshiba Corp.) equipped with low-energy, high-resolution collimators. Around the 140-keV energy peak of ${ }^{99 \mathrm{~m} T c-t e t r o f o s m i n, ~ a ~} 20 \%$ window was used. A total of 90 projections (step-and-shoot mode; $35 \mathrm{~s}$ per projection; imaging time, $23 \mathrm{~min}$ ) were obtained over a $360^{\circ}$ circular orbit, according to standard GMPS imaging protocol in our center. GMPS data acquisition involved 16 frames per cardiac cycle. Data were stored in a $64 \times 64$ matrix. Data were reconstructed by filtered backprojection and then reoriented to yield gated short-axis images. These images were then submitted to the Emory Cardiac Toolbox (Emory University/Syntermed, Atlanta, Ga) for phase analysis (15). The phase of the regional LV count changes throughout the cardiac cycle is an important indicator of the LV function. Because it is related to the time interval when a region in the $3 \mathrm{D} \mathrm{LV}$ myocardial wall starts to contract (presumably, onset of mechanical contraction), it provides information on the uniformity of the distribution of these time intervals for the entire LV-that is, a measure of ventricular synchrony. The phase analysis tool of the Emory Cardiac Toolbox (Emory University/Syntermed) extracts a phase distribution from a gated SPECT study representing the regional LV onset of mechanical contraction in $3 \mathrm{D}$. The phase distribution can be displayed in a polar map or in $3 \mathrm{D}$ and used to generate a phase histogram.

The following 2 quantitative indices were calculated from the phase arrays of all patients: (i) histogram bandwidth, which includes $95 \%$ of the elements of the phase distribution, and (ii) phase SD, which is the SD of the phase distribution (15). From the phase polar maps the site of latest activation was identified (4 regions were evaluated, including anterior, inferior, lateral, and septal). The region with the latest activation had larger phases and appeared in the phase polar map as a brighter region.
In a healthy individual, the ventricle contracts in a coordinated way and most of the myocardial segments have the same or similar phases. Thus, the phase distribution is nearly uniform and the phase histogram is a highly peaked, narrow distribution. As the LV mechanical synchrony worsens, the phase SD and histogram bandwidth are expected to increase.

\section{D Echocardiography}

Two-dimensional echocardiography was performed before CRT implantation and at 6-mo follow-up. Patients were imaged in the left lateral decubitus position with a commercially available system (Vingmed Vivid Seven; GE-Vingmed). Images were acquired in the parasternal view and apical 2- and 4-chamber views using a 3.5-MHz transducer at a depth of $16 \mathrm{~cm}$. Standard 2D and color Doppler data, triggered to the QRS complex, were saved in cineloop format. LV end-systolic volume (LVESV) and LV enddiastolic volume were derived from the conventional apical 2- and 4-chamber views, and LVEF was calculated using the biplane Simpson's rule (17).

\section{CRT Implantation}

For positioning of the LV lead, a coronary sinus venogram was obtained using a balloon catheter, followed by the insertion of the LV pacing lead. An 8-French guiding catheter was used to position the LV lead in the coronary sinus. The preferred position was a lateral or posterolateral vein (18). The right atrial and ventricular leads were positioned conventionally. All leads were connected to a dual-chamber biventricular implantable cardioverter defibrillator (ICD).

\section{Statistical Analysis}

Results are presented as mean \pm SD. Continuous data were compared using the paired or unpaired Student $t$ test when appropriate. Categoric data were compared with the Wilcoxon signed rank test or with the Mann-Whitney test when appropriate. The Fisher exact test was used for proportions. Receiver-operatingcharacteristic (ROC) curves were analyzed to determine the optimal cutoff values of the histogram bandwidth and phase SD to predict response to CRT. For all tests, a $P$ value $<0.05$ was considered statistically significant.

\section{RESULTS}

\section{Study Population}

The baseline characteristics of the 42 patients (31 men, $67 \pm 9$ y) are summarized in Table 1. In total, $28(67 \%)$ patients had ischemic cardiomyopathy, whereas $14(33 \%)$ patients had idiopathic dilated cardiomyopathy. By definition, all patients had severe heart failure (mean NYHA functional class $2.9 \pm 0.4$ ). Thirty-six patients used diuretics $(86 \%), 39$ patients used angiotensin-converting enzyme inhibitors (93\%), and 28 patients used $\beta$-blockers $(67 \%)$. Echocardiographic examination showed a mean LVEDV of $251 \pm 87 \mathrm{~mL}$, a mean LVESV of $193 \pm 83 \mathrm{~mL}$, and a mean LVEF of $24 \% \pm 7 \%$. The mean values for the GMPS variables were as follows: histogram bandwidth, $158^{\circ} \pm$ $65^{\circ}$, and phase SD, $50.8^{\circ} \pm 20.3^{\circ}$. Two patients because of worsening heart failure before the 6-mo follow-up evaluation. 
TABLE 1

Clinical Characteristics of Study Population $(n=42)$

\begin{tabular}{lc}
\hline \multicolumn{1}{c}{ Clinical characteristic } & Value \\
\hline Age (y) & $67 \pm 9$ \\
Men & $31(74)$ \\
Ischemic cardiomyopathy & $28(67)$ \\
Idiopathic dilated cardiomyopathy & $14(33)$ \\
Previous infarction & $24(57)$ \\
Q wave on electrocardiogram & $18(43)$ \\
QRS duration (ms) & $153 \pm 32$ \\
2D echocardiographic variables & \\
LVEDV (mL) & $251 \pm 87$ \\
LVESV (mL) & $193 \pm 83$ \\
LVEF (\%) & $24 \pm 7$ \\
6-min walk test (m) & $330 \pm 109$ \\
Quality-of-life score & $37 \pm 18$ \\
NYHA class & $2.9 \pm 0.4$ \\
& \\
\hline Data are presented as mean \pm SD or as number (\%). \\
\hline
\end{tabular}

\section{CRT Implantation}

CRT device and lead implantation was successful in all patients without major complications (Contak CD or Renewal [Guidant Corp.] or Insync III-CD or Marquis [Medtronic Inc.]). Two types of LV leads were used (Easytrak 4512-80 [Guidant Corp.] or Attain-SD 4189 [Medtronic Inc.]).

\section{Clinical and Echocardiographic Response to CRT}

At the 6-mo follow-up evaluation, the NYHA functional class was significantly improved $(2.9 \pm 0.4$ to $2.0 \pm 0.8[P$ $<0.0001]$ ). The 6-min walking distance increased signif- icantly from $330 \pm 109$ to $395 \pm 117 \mathrm{~m}(P<0.0001)$. In addition, a decrease in the quality-of-life score was demonstrated $(37 \pm 18$ to $22 \pm 19[P<0.0001])$, indicating a general improvement in the quality of life.

Moreover, reverse remodeling was observed on 2D echocardiography: The LVEDV and LVESV decreased significantly (LVEDV from $251 \pm 87$ to $218 \pm 93 \mathrm{~mL}[P<0.001]$ and LVESV from $193 \pm 83$ to $155 \pm 87 \mathrm{~mL}[P<0.0001])$, whereas a significant improvement in LVEF was observed (from $24 \% \pm 7 \%$ to $32 \% \pm 10 \%[P<0.0001]$ ).

\section{Responders and Nonresponders}

After the 6-mo follow-up, 30 (71\%) patients were classified as responders, according to an improvement of $\geq 1$ NYHA functional class. On the other hand, 12 (29\%) patients, of whom 2 died of worsening heart failure before the 6-mo follow-up, did not improve $\geq 1$ NYHA functional class and were, therefore, classified as nonresponders.

At baseline, there were no significant differences in clinical characteristics between the responders and nonresponders (Table 2), except for the histogram bandwidth $\left(175^{\circ} \pm 63^{\circ}\right.$ vs. $\left.117^{\circ} \pm 51^{\circ}[P<0.01]\right)$ and phase SD $\left(56.3^{\circ} \pm 19.9^{\circ}\right.$ vs. $\left.37.1^{\circ} \pm 14.4^{\circ}[P<0.01]\right)$, which were significantly larger in responders as compared with nonresponders.

The nonresponders tended to have smaller LV volumes at baseline, although these differences were not statistically significant. However, a statistically significant difference in the LV dyssynchrony variables derived from GMPS was observed.

In the responders, the mean NYHA functional class improved from $2.9 \pm 0.3$ to $1.7 \pm 0.5$, whereas it remained unchanged in the nonresponders $(2.8 \pm 0.6$ vs. $3.0 \pm 0.7$, by definition).

TABLE 2

Baseline Characteristics of Responders ( $n=30)$ vs. Nonresponders $(n=12)$ to CRT

\begin{tabular}{|c|c|c|c|}
\hline Baseline characteristic & Responders & Nonresponders & $P$ value \\
\hline Age (y) & $67 \pm 8$ & $66 \pm 11$ & NS \\
\hline Men & $22(73)$ & $9(75)$ & NS \\
\hline Ischemic cardiomyopathy & $19(63)$ & $9(75)$ & NS \\
\hline Idiopathic dilated cardiomyopathy & $11(37)$ & $3(25)$ & NS \\
\hline Previous infarction & $17(57)$ & $7(58)$ & NS \\
\hline Q wave on electrocardiogram & $11(37)$ & $7(58)$ & NS \\
\hline \multicolumn{4}{|l|}{ 2D echocardiographic variables } \\
\hline LVEDV (mL) & $267 \pm 88$ & $212 \pm 74$ & NS \\
\hline LVESV (mL) & $209 \pm 88$ & $154 \pm 55$ & NS \\
\hline LVEF (\%) & $23 \pm 7$ & $27 \pm 8$ & NS \\
\hline 6 -min walk test $(\mathrm{m})$ & $345 \pm 104$ & $289 \pm 117$ & NS \\
\hline Quality-of-life score & $35 \pm 17$ & $43 \pm 18$ & NS \\
\hline NYHA class & $2.9 \pm 0.3$ & $2.8 \pm 0.6$ & NS \\
\hline \multicolumn{4}{|l|}{ GMPS variables } \\
\hline Histogram bandwidth $\left(^{\circ}\right)$ & $175 \pm 63$ & $117 \pm 51$ & $<0.01$ \\
\hline Phase SD $\left(^{\circ}\right)$ & $56.3 \pm 19.9$ & $37.1 \pm 14.4$ & $<0.01$ \\
\hline $\begin{array}{l}\mathrm{NS}=\text { not statistically significant. } \\
\text { Data are presented as mean } \pm \mathrm{S}\end{array}$ & & & \\
\hline
\end{tabular}


The quality-of-life score improved from $35 \pm 17$ to $17 \pm$ $16(P<0.0001)$, and the 6-min walking distance increased from $345 \pm 104$ to $428 \pm 97 \mathrm{~m}(P<0.0001)$ in the responder group, whereas no improvement in the qualityof-life score $(43 \pm 18$ to $41 \pm 18[P=$ not statistically significant $[\mathrm{NS}])$ or in 6-min walking distance $(289 \pm 117$ to $284 \pm 116 \mathrm{~m}[P=\mathrm{NS}])$ was noted in the nonresponders.

Moreover, a significant improvement in the echocardiographic variables was observed in the responders (Fig. 1). In particular, LVEDV decreased from $267 \pm 88$ to $225 \pm$ $102 \mathrm{~mL}(P<0.0001)$ and LVESV decreased from $209 \pm$ 88 to $159 \pm 97 \mathrm{~mL}(P<0.0001)$; the LVEF increased significantly from $23 \% \pm 7 \%$ to $33 \% \pm 11 \%(P<0.0001)$. In the nonresponders, the LVEF and LV volumes remained unchanged (Table 2; Fig. 1). An example of phase analysis in a nonresponder is shown in Figure 2A, whereas an example of phase analysis in a responder to CRT is provided in Figure 2B.

\section{Histogram Bandwidth and Phase SD for Prediction of Response to CRT}

The only 2 variables at baseline that were significantly different between responders and nonresponders were the histogram bandwidth and the phase SD.

To define the optimal cutoff value for histogram bandwidth and phase SD to predict response to CRT, ROC curve analysis was performed. When defining responders as patients exhibiting an improvement in NYHA functional class of $\geq 1$ score, an optimal sensitivity and specificity of $70 \%$ was obtained at a cutoff value of $135^{\circ}$ (Fig. 3). The area under the curve was 0.78 , which indicates good predictive value. ROC curve analysis was also performed to define the optimal cutoff value for phase SD to predict response to CRT. The area under the curve was 0.81 , which also indicates good predictive value for the response to CRT. A cutoff value of $43^{\circ}$ yielded a sensitivity and specificity of $74 \%$ to predict response to CRT (Fig. 4).

\section{DISCUSSION}

The results of the current study demonstrate that LV dyssynchrony assessed with phase analysis from GMPS is related to the clinical response to CRT. Except for the histogram bandwidth and the phase SD, all other baseline characteristics were comparable between the nonresponders and the responders to CRT. A cutoff value of $135^{\circ}$ for histogram bandwidth can predict the clinical response to CRT with a sensitivity and specificity of $70 \%$, whereas a cutoff value of $43^{\circ}$ for phase SD yielded a sensitivity and specificity of $74 \%$ for prediction of the clinical response to CRT.

Heart failure is an important health care problem in the Western world, and the 5-y mortality exceeds 50\% $(19,20)$. CRT has been introduced as an adjuvant therapy for patients with end-stage heart failure, and promising results have been reported. Not only has acute improvement in hemodynamics been observed after CRT implantation, but
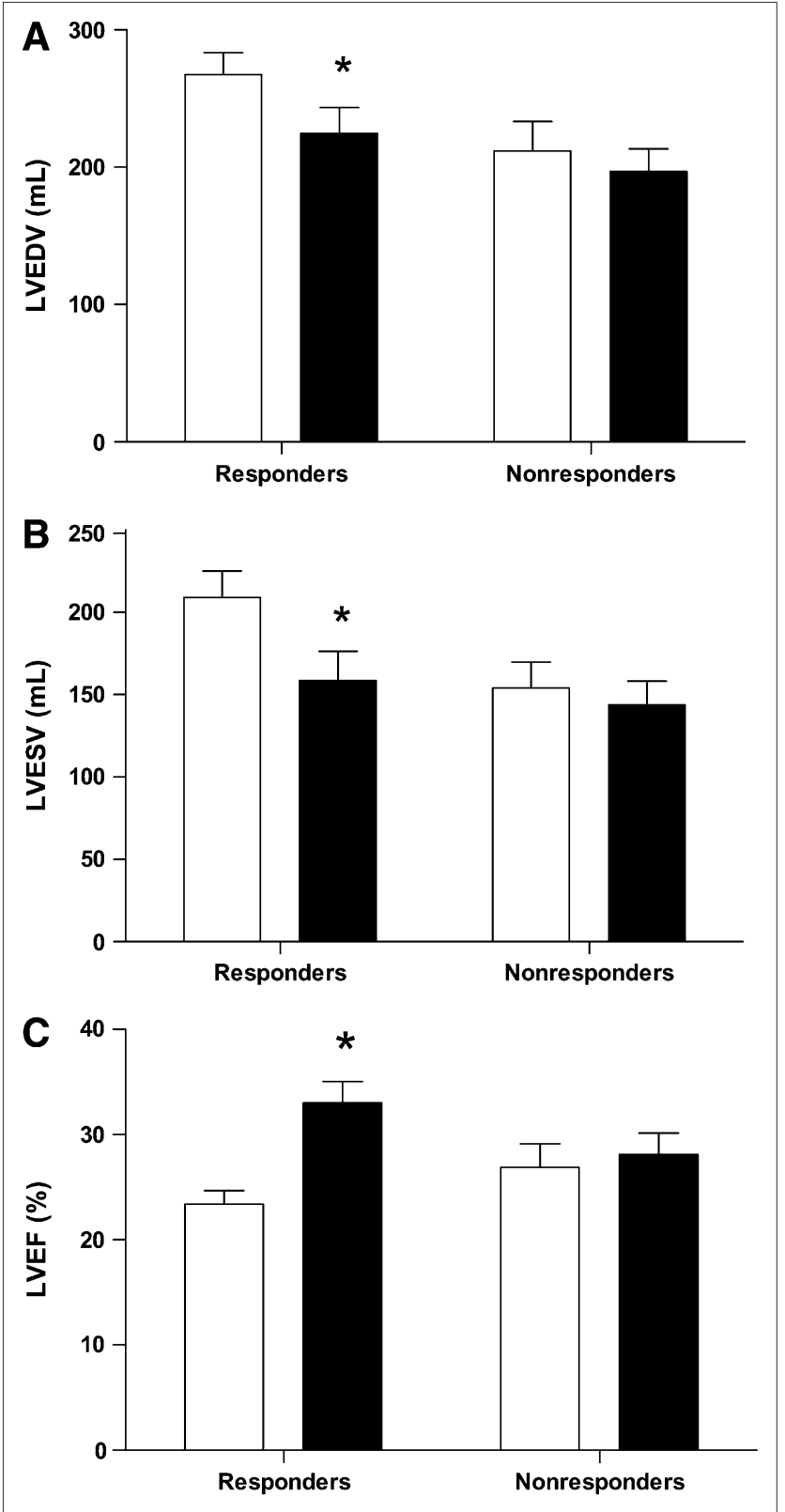

FIGURE 1. (A) Changes in LVEDV from baseline (white bars) to 6-mo follow-up (black bars) in responders and nonresponders to CRT. LVEDV decreased significantly in responders and remained unchanged in nonresponders. ${ }^{*} P<0.0001$ baseline vs. 6-mo follow-up. (B) Changes in LVESV from baseline (white bars) to 6-mo follow-up (black bars) in responders and nonresponders to CRT. LVESV decreased significantly in responders and remained unchanged in nonresponders. ${ }^{*} P<$ 0.0001 baseline vs. 6-mo follow-up. (C) Changes in LVEF from baseline (white bars) to 6-mo follow-up (black bars) in responders and nonresponders to CRT. LVEF increased significantly in responders and remained unchanged in nonresponders. ${ }^{*} P<0.0001$ baseline vs. 6 -mo follow-up.

also sustained clinical benefit (improvement in quality of life, exercise capacity, heart failure symptoms, and LV systolic function) at longer follow-up (1,2,21-23). However, it has been noted that approximately $20 \%-30 \%$ of the patients do not respond to CRT (21), and it has been 
A
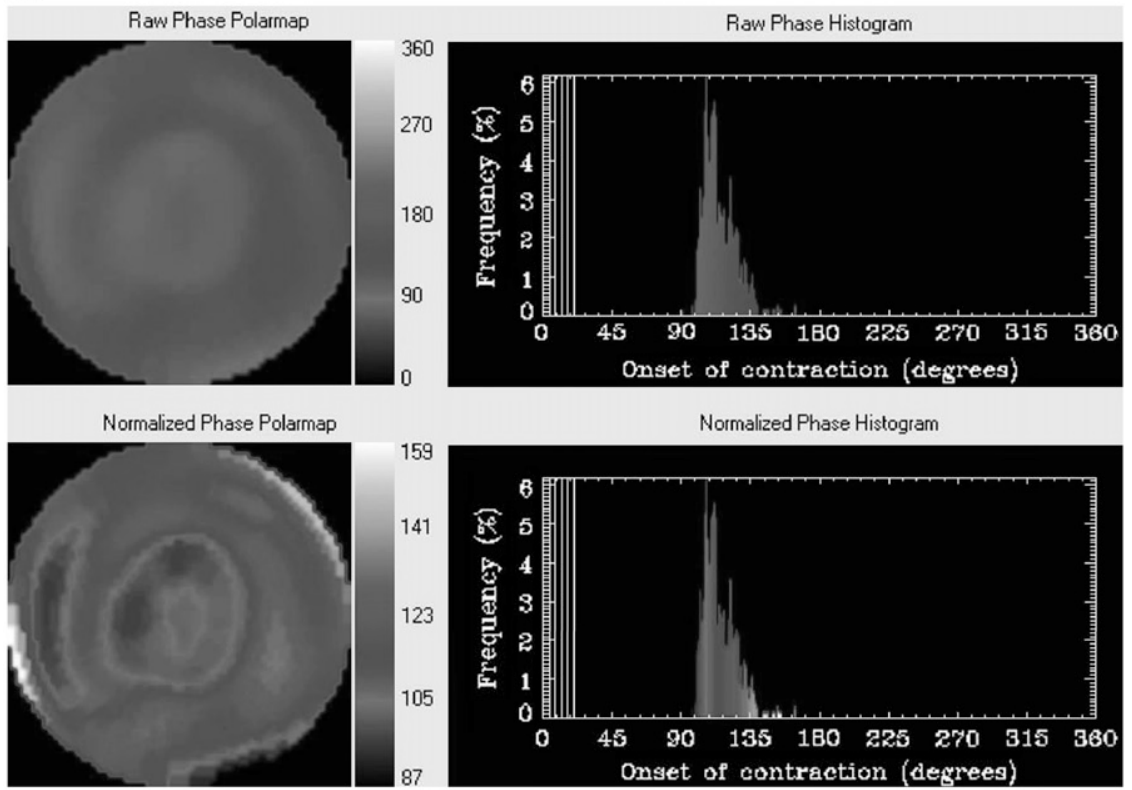

FIGURE 2. (A) Example of phase analysis in a nonresponder to CRT. At baseline, no LV dyssynchrony with phase analysis was present in this patient. Nonnormalized (top panel) and normalized (bottom panel) phase distributions are relatively uniform and the corresponding phase histograms are highly peaked, narrow distributions. After 6-mo follow-up, no response to CRT was observed, as reflected by deterioration in NYHA functional class from 3 to 4 . In addition, LVEF remained unchanged (baseline [32\%] vs. 6-mo follow-up [33\%]). (B) Example of phase analysis in a responder to CRT. In this patient, LV dyssynchrony with phase analysis was present at baseline. Nonnormalized (top panel) and normalized (bottom panel) phase distributions show substantial nonuniformity, whereas the corresponding phase histograms are widely spread distributions. After 6-mo follow-up, this patient improved in NYHA functional class from 3 to 2, indicating response to CRT. In addition, LVEF increased from $27 \%$ at baseline to $33 \%$ at 6 -mo followup.
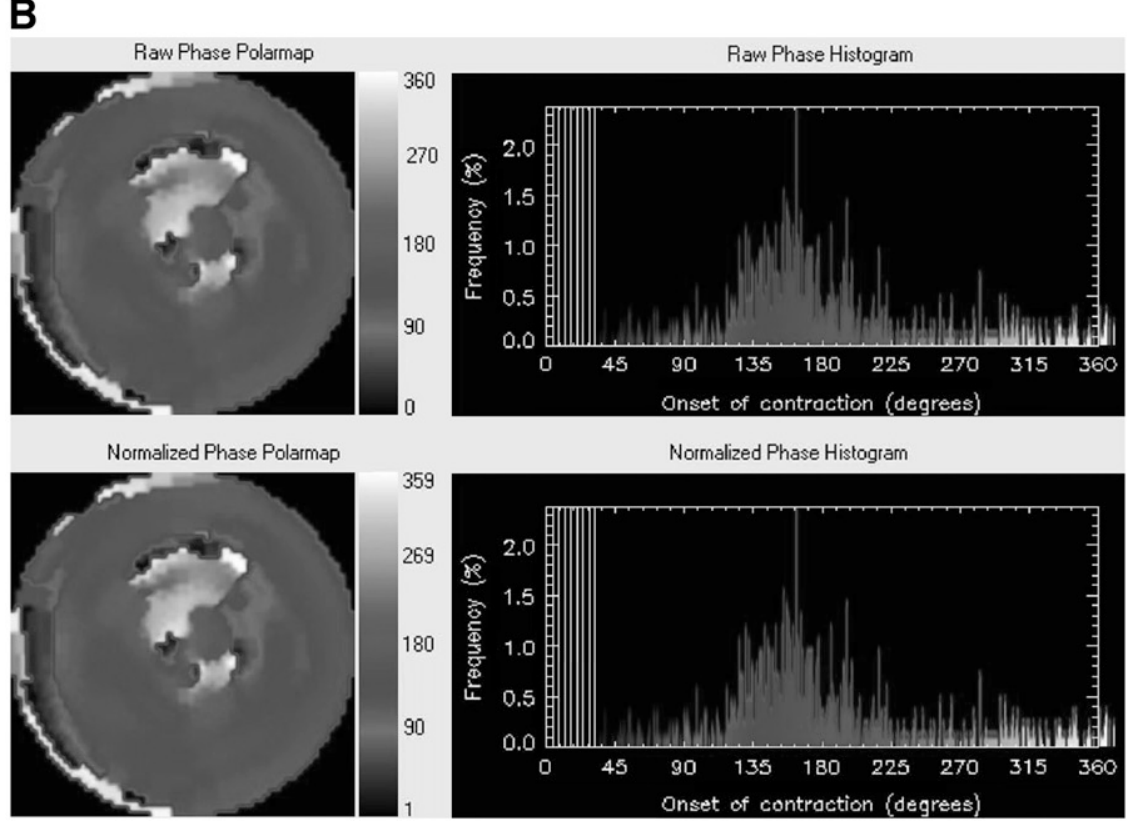

demonstrated that LV dyssynchrony assessed with TDI can predict the clinical response to CRT with a sensitivity and specificity of $80 \%$ (5). Nuclear imaging techniques have been applied since the early 1980s for the evaluation of inter- and intraventricular dyssynchrony. Fauchier et al. (24) evaluated the prognostic value of inter- and intraventricular dyssynchrony in 103 patients with idiopathic dilated cardiomyopathy with equilibrium radionuclide angiography and Fourier phase analysis. It was shown that the intraventricular dyssynchrony was the only independent predictor of cardiac events.

The long-term effects of biventricular pacing have been investigated by Toussaint et al. (25). The authors evaluated
34 patients with radionuclide ventriculography. Patients were evaluated before CRT implantation, $8 \mathrm{~d}$ after implantation, and thereafter every 6 mo. It was noted that biventricular pacing reduced the electrical and mechanical dyssynchrony, associated with a significant improvement in LV function.

Tsurugaya et al. (26) have reported on quantitative gated SPECT before and after CRT in 10 heart failure patients. At a follow-up of $18 \pm 6$ mo, patients were classified as responders or nonresponders according to their clinical status and echocardiographic parameters. A significant improvement in LV synchrony was observed in the responders to CRT, whereas no improvement could be demonstrated in 


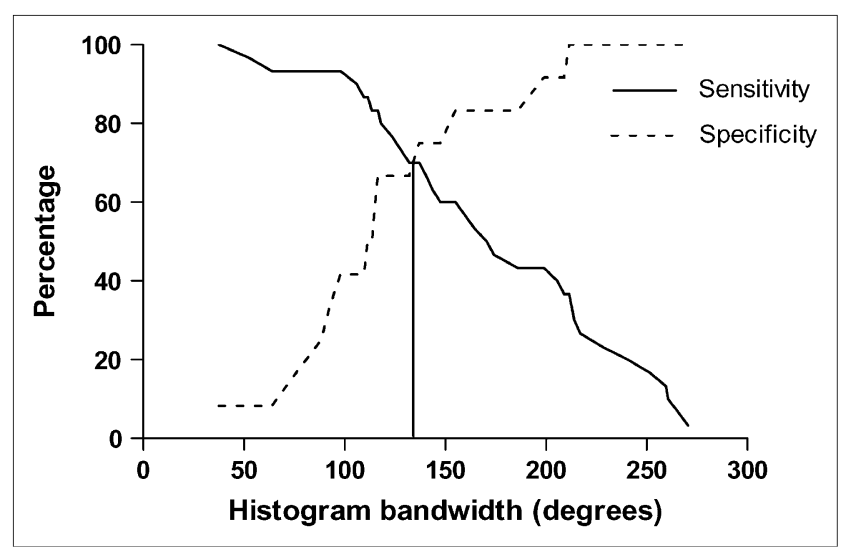

FIGURE 3. ROC analysis of histogram bandwidth demonstrated a sensitivity and specificity of $70 \%$ to predict response to CRT at cutoff level of $135^{\circ}$ for histogram bandwidth.

the nonresponders. Sciagrà et al. (27) investigated the use of gated perfusion SPECT for baseline evaluation and follow-up of patients referred for CRT. The authors showed that patients with extensive resting perfusion defects had limited response to CRT. Moreover, no significant reduction in LV volumes or improvement in LVEF could be observed in patients with extensive perfusion defects, whereas a significant improvement in LV systolic function and LV volumes was noted in patients without extensive perfusion defects.

Recently, a new technique has been developed to evaluate LV dyssynchrony with phase analysis from GMPS (15). The fully automated analysis has been shown to yield highly reproducible results, and the method can even be applied to severely hypoperfused myocardium (28). The information of LV dyssynchrony can be obtained from the same GMPS dataset as used for the evaluation of cardiac function and perfusion.

We have recently reported on the correlation between the different quantitative indices obtained by phase analysis with GMPS and the LV dyssynchrony as assessed with TDI

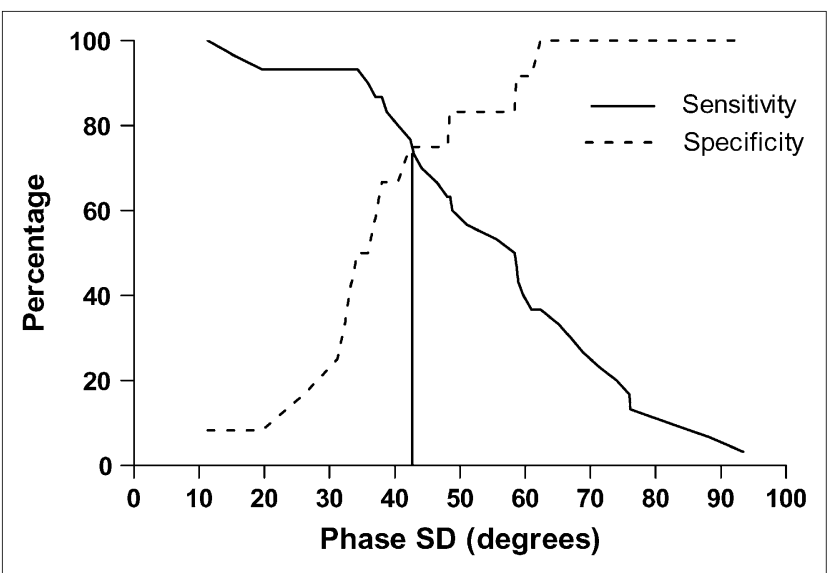

FIGURE 4. ROC curve analysis of phase SD demonstrated a sensitivity and specificity of $74 \%$ to predict response to CRT at cutoff level of $43^{\circ}$ for phase SD. in 75 patients with heart failure (29). The results indicated good correlations between TDI and histogram bandwidth/ phase SD for assessment of LV dyssynchrony.

In the current study, 42 patients were included who underwent CRT implantation to evaluate whether the degree of LV dyssynchrony as assessed with GMPS could predict which patients will respond to CRT. In this study, the use of phase analysis from GMPS has been applied, to our knowledge for the first time, to predict response to CRT, yielding a sensitivity and specificity of $70 \%$ for histogram bandwidth and a sensitivity and specificity of $74 \%$ for phase SD to predict response to CRT.

Several limitations of this study must be considered. First, our research involved a relatively small study population, and the results must be confirmed in larger prospective patient populations. A more general limitation of GMPS is the radiation burden, which makes this technique less suitable for follow-up studies. However, considering the clinical circumstances of this specific patient population and the potential risk-to-benefit ratio in these patients, follow-up with GMPS could be justified in selected cases after CRT implantation. Also, follow-up studies with GMPS can provide valuable information on functional variables, including LV volumes and function. Also, the temporal resolution that gated-SPECT MPI provides is perceived to be relatively low compared with echocardiography. Nevertheless, it must be noted that the phase analysis tool uses the first harmonic Fourier approximation that can enhance its phase calculation when applied to the data with lower temporal resolution. It transforms the discrete data points into a continuous curve. Only the systolic portion of the data is used to determine the phase. By having this curve fit closely with the systolic data points, the artifactual phase difference resulting from low temporal resolution is greatly reduced. Figure 5 shows the small difference of the phases obtained from a patient when data are acquired at 8 frames per cardiac cycle compared with when data are acquired at 16 frames per cardiac cycle. This is consistent with previous work showing the value of Fourier temporal interpolation of data acquired at 8 frames per cycle by improving image quality without detrimental effects of quantitative parameters such as ejection fraction and left ventricular volumes (30).

Recent studies have reported the presence of perfusion defects or scar tissue and the influence on the response to CRT $(27,31)$. The aim of our study was to determine whether the presence of LV dyssynchrony, as assessed with phase analysis on GMPS, can predict response to CRT, and the influence of scar tissue (or perfusion defects) was not included in the current analysis.

However, other techniques also have important disadvantages. MRI is less suitable for patients with pacemakers and ICDs, and the prevalence of claustrophobia disqualifies another important percentage of the patients. TDI with echocardiography is highly operator dependent, and 20\% of patients have a suboptimal acoustic window. Moreover, 
FIGURE 5. Comparison of difference of phases obtained when 8 frames per cycle (left panel) or 16 frames per cycle (right panel) data acquisition are used. Points in right panel are counts of a pixel arbitrarily chosen from the anterior region of polar maps given by a set of gated (16 frames per cycle) short-axis images. These points are regional maximum counts and their variations represent wall thickness changes at region over the cardiac cycle. Points in left panel are down-sampled from points in right panel. Curves are the first harmonics that approximate wall thickness changes during the cardiac cycle. With first harmonic approximation, phase difference between 8 frames per cycle and 16 frames per cycle is very small at $-0.5^{\circ}$ $\left(360^{\circ}\right.$ corresponds to 1 cardiac cycle).

the phase analysis from GMPS has a major advantage in that this approach permits integrated assessment of LV function, perfusion (to identify ischemia and scar tissue), and dyssynchrony. All of this information may be of use in determining whether to implant a CRT device $(32,33)$.

\section{CONCLUSION}

The clinical response to CRT is related to the presence of LV dyssynchrony assessed by phase analysis from GMPS. Both histogram bandwidth and phase SD can be used to predict response to CRT, with sensitivity and specificity of $70 \%$ and $74 \%$, respectively. The current results need confirmation in larger patient populations.

\section{ACKNOWLEDGMENTS}

Ernest V. Garcia receives royalties from the sale of the Emory Cardiac Toolbox. The terms of this arrangement have been reviewed and approved by Emory University in accordance with its conflict-of-interest practice. The other authors have indicated they have no financial or other conflicts of interest. Jeroen J. Bax has research grants from Guidant, Medtronic, GE Healthcare, and BMS Medical Imaging. Gabe B. Bleeker is supported by the Dutch Heart Foundation (grant 2002B109).

\section{REFERENCES}

1. Leclercq C, Kass DA. Retiming the failing heart: principles and current clinical status of cardiac resynchronization. J Am Coll Cardiol. 2002;39:194-201.

2. Abraham WT, Hayes DL. Cardiac resynchronization therapy for heart failure. Circulation. 2003;108:2596-2603.

3. Leclercq C, Hare JM. Ventricular resynchronization: current state of the art. Circulation. 2004;109:296-299.

4. Auricchio A, Stellbrink C, Block M, et al. Effect of pacing chamber and atrioventricular delay on acute systolic function of paced patients with congestive heart failure: the Pacing Therapies for Congestive Heart Failure Study Group-The Guidant Congestive Heart Failure Research Group. Circulation. 1999;99:2993-3001.
5. Bax JJ, Bleeker GB, Marwick TH, et al. Left ventricular dyssynchrony predicts response and prognosis after cardiac resynchronization therapy. J Am Coll Cardiol. 2004;44:1834-1840.

6. Bax JJ, Marwick TH, Molhoek SG, et al. Left ventricular dyssynchrony predicts benefit of cardiac resynchronization therapy in patients with end-stage heart failure before pacemaker implantation. Am J Cardiol. 2003;92:1238-1240.

7. Bleeker GB, Schalij MJ, Molhoek SG, et al. Relationship between QRS duration and left ventricular dyssynchrony in patients with end-stage heart failure. J Cardiovasc Electrophysiol. 2004;15:544-549.

8. Yu CM, Lin H, Zhang Q, Sanderson JE. High prevalence of left ventricular systolic and diastolic asynchrony in patients with congestive heart failure and normal QRS duration. Heart. 2003;89:54-60.

9. Suffoletto MS, Dohi K, Cannesson M, Saba S, Gorcsan J III. Novel speckletracking radial strain from routine black-and-white echocardiographic images to quantify dyssynchrony and predict response to cardiac resynchronization therapy. Circulation. 2006;113:960-968.

10. Westenberg JJ, Lamb HJ, van der Geest RJ, et al. Assessment of left ventricular dyssynchrony in patients with conduction delay and idiopathic dilated cardiomyopathy: head-to-head comparison between tissue Doppler imaging and velocity-encoded magnetic resonance imaging. J Am Coll Cardiol. 2006;47: 2042-2048.

11. Wackers FJ, Berger HJ, Johnstone DE, et al. Multiple gated cardiac blood pool imaging for left ventricular ejection fraction: validation of the technique and assessment of variability. Am J Cardiol. 1979;43:1159-1166.

12. Upton MT, Rerych SK, Newman GE, Bounous EP Jr, Jones RH. The reproducibility of radionuclide angiographic measurements of left ventricular function in normal subjects at rest and during exercise. Circulation. 1980;62: 126-132.

13. Berman DS, Germano G. Evaluation of ventricular ejection fraction, wall motion, wall thickening, and other parameters with gated myocardial perfusion single-photon emission computed tomography. J Nucl Cardiol. 1997;4:S169S171.

14. Levine MG, McGill CC, Ahlberg AW, et al. Functional assessment with electrocardiographic gated single-photon emission computed tomography improves the ability of technetium-99m sestamibi myocardial perfusion imaging to predict myocardial viability in patients undergoing revascularization. Am J Cardiol. 1999;83:1-5.

15. Chen J, Garcia EV, Folks RD, et al. Onset of left ventricular mechanical contraction as determined by phase analysis of ECG-gated myocardial perfusion SPECT imaging: development of a diagnostic tool for assessment of cardiac mechanical dyssynchrony. J Nucl Cardiol. 2005;12:687-695.

16. Rector TS, Kubo SH, Cohn JN. Validity of the Minnesota Living with Heart Failure questionnaire as a measure of therapeutic response to enalapril or placebo. Am J Cardiol. 1993;71:1106-1107.

17. Schiller NB, Acquatella H, Ports TA, et al. Left ventricular volume from paired biplane two-dimensional echocardiography. Circulation. 1979;60:547-555.

18. Alonso C, Leclercq C, Victor F, et al. Electrocardiographic predictive factors of long-term clinical improvement with multisite biventricular pacing in advanced heart failure. Am J Cardiol. 1999;84:1417-1421.

19. Khand A, Gemmel I, Clark AL, Cleland JG. Is the prognosis of heart failure improving? J Am Coll Cardiol. 2000;36:2284-2286. 
20. Levy D, Kenchaiah S, Larson MG, et al. Long-term trends in the incidence of and survival with heart failure. N Engl J Med. 2002;347:1397-1402.

21. Abraham WT, Fisher WG, Smith AL, et al. Cardiac resynchronization in chronic heart failure. N Engl J Med. 2002;346:1845-1853.

22. Cazeau S, Leclercq C, Lavergne T, et al. Effects of multisite biventricular pacing in patients with heart failure and intraventricular conduction delay. $N$ Engl J Med. 2001;344:873-880.

23. Auricchio A, Stellbrink C, Sack S, et al. Long-term clinical effect of hemodynamically optimized cardiac resynchronization therapy in patients with heart failure and ventricular conduction delay. J Am Coll Cardiol. 2002;39:20262033.

24. Fauchier L, Marie O, Casset-Senon D, Babuty D, Cosnay P, Fauchier JP. Interventricular and intraventricular dyssynchrony in idiopathic dilated cardiomyopathy: a prognostic study with Fourier phase analysis of radionuclide angioscintigraphy. J Am Coll Cardiol. 2002;40:2022-2030.

25. Toussaint JF, Lavergne T, Kerrou K, et al. Basal asynchrony and resynchronization with biventricular pacing predict long-term improvement of LV function in heart failure patients. Pacing Clin Electrophysiol. 2003;26:1815-1823.

26. Tsurugaya $\mathrm{H}$, Tada $\mathrm{H}$, Toyama $\mathrm{T}$, et al. Usefulness of quantitative gate singlephoton emission computed tomography to evaluate ventricular synchrony in patients receiving biventricular pacing. Am J Cardiol. 2004;94:127-130.
27. Sciagrà R, Giaccardi M, Porciani MC, et al. Myocardial perfusion imaging using gated SPECT in heart failure patients undergoing cardiac resynchronization therapy. J Nucl Med. 2004;45:164-168.

28. Cooke CD, Garcia EV, Cullom SJ, Faber TL, Pettigrew RI. Determining the accuracy of calculating systolic wall thickening using a fast Fourier transform approximation: a simulation study based on canine and patient data. J Nucl Med. 1994;35:1185-1192.

29. Henneman MM, Chen J, Ypenburg C, et al. Phase analysis of gated myocardial perfusion SPECT compared to tissue Doppler imaging for the assessment of left ventricular dyssynchrony. J Am Coll Cardiol. 2007;49:1708-1714.

30. Gremillet E, Champailler A, Soler C. Fourier temporal interpolation improves electrocardiograph-gated myocardial perfusion SPECT. J Nucl Med. 2005;46: 1769-1774.

31. Ypenburg C, Schalij MJ, Bleeker GB, et al. Impact of viability and scar tissue on response to cardiac resynchronization therapy in ischaemic heart failure patients. Eur Heart J. 2007;28:33-41.

32. Bax JJ, Abraham T, Barold SS, et al. Cardiac resynchronization therapy. Part 1. issues before device implantation. J Am Coll Cardiol. 2005;46:2153-2167.

33. Bax JJ, Abraham T, Barold SS, et al. Cardiac resynchronization therapy. Part 2. issues during and after device implantation and unresolved questions. J Am Coll Cardiol. 2005;46:2168-2182 